\title{
Ion acoustic solitary waves with adiabatic ions in magnetized electron-positron-ion plasmas
}

\author{
S. Mahmood ${ }^{\mathrm{a}}$ and N. Akhtar ${ }^{\mathrm{b}}$ \\ Theoretical Plasma Physics Division, Pinstech, P.O. Nilore, Islamabad, Pakistan
}

Received 28 May 2008 / Received in final form 31 July 2008

Published online 27 August 2008 - (c) EDP Sciences, Società Italiana di Fisica, Springer-Verlag 2008

\begin{abstract}
Linear and nonlinear ion acoustic waves in the presence of adiabatically heated ions in magnetized electron-positron-ion plasmas are studied. The Sagdeev potential approach is employed to obtain the energy integral equation in such a mulitcomponent plasma using fluid theory. It is found that electron density humps are formed in the subsonic region in magnetized electron-positron-ion plasmas. The amplitude of electron density hump is decreased with the increase of hot ion temperature in electron-positron-ion plasmas. However, the increase in positron concentration and obliqueness of the wave increases the amplitude of nonlinear structure. The increase in positron concentration also reduces the width of the nonlinear structure in a magnetized multicomponent plasma. The numerical solutions in the form of solitary pulses are also presented for different plasma cases. The results may be applicable to astrophysical plasma situations, where magnetized electron-positron-ion plasma with hot ions can exist.
\end{abstract}

PACS. 52.27.Cm Multicomponent and negative-ion plasmas - 52.25.Xz Magnetized plasmas - 52.35.Fp Electrostatic waves and oscillations - 52.35.Sb Solitons; BGK modes

\section{Introduction}

The electron-positron (e-p) plasma is the major constituent in the pulsar magnetosphere $[1,2]$, relativistic jets that streams from the nuclei of quasars and active galactic nuclei (AGN) [3]. It is believed that around such astrophysical e-p plasma situations, the possibility of existence of ions cannot be ignored. The dynamic scales of e-p plasma changes drastically with the inclusion of ions, which are massive particles than same mass particles i.e., electrons and positrons. For example, ion acoustic phenomenon can occur in e-p plasmas only with the inclusion of ions which does not exist in pure e-p (pair) plasmas. The linear wave spectrum of e-p plasmas also increases with the inclusion of ions and wide range of frequencies are available in e-p-i plasmas as compared to two component e-p or e-i (electron-ion) plasmas. The possibility of wave-wave interaction increases and more interesting nonlinear phenomenon could happen in electron-positronion (e-p-i) plasmas. In most of the astrophysical plasmas situations, the kinetic energy of electron (positron) is much greater than the rest mass energy of the electron (positron). Therefore, most of the theoretical investigations of e-p and e-p-i plasmas have been done in the rel-

\footnotetext{
${ }^{a}$ e-mail: shahzadm100@gmail.com

b Present address: Max-Planck-Institut für Sonnensystemforschung, Max-Planck-Str. 2, 37191 Katlenburg-Lindau, Germany
}

ativistic regime [4-6]. However, the study of nonrelativistic astrophysical e-p and e-p-i plasmas is also important because e-p plasmas can radiate energy very effectively through cyclotron emission and cool down eventually $[7,8]$. A number of experiments have been proposed for e-p plasma studies in the laboratory [7]. The positron can be used to study the plasma transport mechanism in tokamaks $[9,10]$. The positrons can be introduce in tokamaks by injecting burst of neutral positronium $\left(\mathrm{e}^{+} \mathrm{e}^{-}\right)$atoms, which are then ionized by plasmas. The annihilation time of these positrons in the plasma is long compared to the typical particle confinement time in tokamaks. Therefore, study of linear and nonlinear waves in e-p-i plasmas becomes interesting to understand some aspects of dynamic behaviour of both astrophysical and laboratory multicomponent plasmas. It is believed that very strong magnetic field $\sim 10^{12} \mathrm{G}$ can exist near magnetic poles of the pulsar and plasma number density upto the order of $10^{14}$ or $10^{15} \mathrm{~cm}^{-3}$ may be present there, which consists of positrons and electrons with relativistic energies [11]. However there is still possibility of existence of protons around the atmosphere of pulsar magnetosphere.

The linear and nonlinear ion acoustic waves in unmagnetized [12-14] and magnetized [15,16] electron-positronion (e-p-i) plasmas have already been studied. Most of these studies have been done with the assumption of cold ions in such a multicomponent plasma. Nejoh [14] investigated ion acoustic solitary waves in a unmagnetized 
e-p-i plasma case with hot ions. The ions are taken to be dynamic and adiabatically heated while the electrons and positrons are assumed to be isothermal and follow Boltzmann distribution. However, most of the e-p-i astrophysical plasma situations are magnetized and ions can also be hot in those multicomponent plasma regions. Therefore, it will be interesting to study arbitrary amplitude ion acoustic solitary waves in the presence of hot ions in magnetized e-p-i plasmas. The consistency of fluid theory in the presence of hot and dynamic ions in plasmas demands $c_{s} \gg v_{t i}$ (where $c_{s}=\sqrt{T_{e} / m_{i}}$ is the ion acoustic speed and $v_{t i}=\sqrt{T_{i} / m_{i}}$ is the ion thermal speed) and Landau damping effects are ignored in the model. The ion acoustic solitary waves in magnetized electron-ion (e-i) plasmas with cold ions $[17,18]$ as well as adiabatic ions [19] have also been investigated.

In this manuscript, the propagation of ion acoustic solitary wave in a magnetized homogeneous e-p-i plasma in the presence of adiabatically heated ions are studied. In Section 2, the nonlinear set of equations and dispersion relation for low frequency ion acoustic waves are presented. In Section 3, the energy integral equation is obtained with Sagdeev potential approach. Some of the possible numerical solutions have also been presented in Section 4. Finally in Section 5, the conclusion is presented.

\section{Nonlinear set of equations}

Let us consider an ideal homogeneous magnetized three component (e-p-i) plasma. The external magnetic field is directed along $z$-axis i.e., $\mathbf{B}_{0}=B_{0} \hat{z}$. The electrons and positrons are assumed to be isothermal and Boltzmann distributed while the ions are taken to be dynamic and adiabatically heated. The phase velocity of the IAW is assumed much less than the electron (positron) thermal velocities i.e., $\frac{\omega}{k} \ll v_{t e}, v_{t p}$ (where $v_{t j}=\left(\frac{T_{j}}{m_{j}}\right)^{\frac{1}{2}}$ is the thermal speed of the $j$ th species while $j=\mathrm{e}, \mathrm{p}, \mathrm{i})$. It is also assumed that $T_{i} \ll T_{e}$ for the consistency of the fluid model. Under these conditions the nonlinear dynamics of the low frequency IAW in the three component plasma are governed by the following set of equations.

The ion continuity equation is described as,

$$
\frac{\partial n_{i}}{\partial t}+\nabla \cdot\left(n_{i} \mathbf{v}_{i}\right)=0
$$

The ion momentum equation is given as,

$$
\frac{\partial \mathbf{v}_{i}}{\partial t}+\left(\mathbf{v}_{i} . \nabla\right) \mathbf{v}_{i}=-\frac{e}{m_{i}} \nabla \phi+\omega_{c i} \mathbf{v}_{i} \hat{z}-\frac{1}{m_{i} n_{i}} \nabla \tilde{P}_{i}
$$

where pressure tensor is defined as follows,

$$
\tilde{P}_{i}=p_{\perp i} \hat{I}+\left(p_{\| i}-p_{\perp i}\right) \hat{z} \hat{z}
$$

here $\hat{I}$ is the unit tensor and $\hat{z}$ is the unit vector along the external magnetic field. The parallel and perpendicular pressure components are represented as $p_{\| i}$ and $p_{\perp i}$ respectively. In case of isotropic ion pressure, we have $p_{\| i}=p_{\perp i}\left(=p_{i}\right.$ say $)$ and therefore $\nabla \tilde{P}_{i}=\nabla p_{i}$. For adiabatic ion pressure, we have,

$$
p_{i}=p_{i 0}\left(\frac{n_{i}}{n_{i 0}}\right)^{\gamma},
$$

where $\gamma=\frac{(N+2)}{N}$, and $N$ is the number of degrees of freedom. In case of magnetized plasmas, we have $N=3$ hence $\gamma=\frac{5}{3}$ and equilibrium ion pressure is defined as $p_{i 0}=n_{i 0} T_{i}$.

At low frequency waves, the electrons and positrons (which have the same mass) are more magnetized as compared to ions because ions are much heavier than electrons (i.e., ion mass is $\sim 10^{3}$ times greater than the mass of electron or positron). Therefore ion Larmor radius is much larger than the Larmor radius of electron or positron. Therefore electrons and positrons are assumed to move almost parallel to external magnetic field as compared to ions. Under this assumption, the electrons and positrons in the electrostatic potential perturbations follow the Boltzmann distributions, respectively as,

$$
n_{e}=n_{e 0} e^{\frac{e \phi}{T_{e}}}
$$

and

$$
n_{p}=n_{p 0} e^{\frac{-e \phi}{T p}} .
$$

The Poisson equation gives,

$$
\nabla^{2} \phi=-4 \pi e\left(n_{i}+n_{p}-n_{e}\right) .
$$

We have assumed $\mathbf{E}=-\nabla \phi$ (where $\phi$ is an electrostatic potential) and $\omega_{c i}=\left(\frac{e B_{0}}{m_{i} c}\right)$ is the ion gyrofrequency, while $n_{j}$ is the perturbed density of $j$ th species. Here $n_{j 0}$ is the unperturbed density of the species (where $j=\mathrm{e}, \mathrm{p}, \mathrm{i}$ stands for electrons, positrons and ions, respectively), $v_{i}$ is the ion fluid velocity, $e$ is the magnitude of the electron charge and $m_{i}$ is the mass of the ion and $T_{e}\left(T_{p}, T_{i}\right)$ denote the electron (positron, ion) temperatures, respectively.

In equilibrium, we have

$$
n_{i 0}+n_{p 0}=n_{e 0}
$$

Let us consider the two dimensional perturbation in the $x z$-plane, so that $\nabla=\left(\partial_{x}, 0, \partial_{z}\right)$. Then equations (1) and (2) can be written as,

$$
\partial_{t} n_{i}+\partial_{x}\left(n_{i} v_{i x}\right)+\partial_{z}\left(n_{i} v_{i z}\right)=0
$$

$$
\begin{gathered}
\partial_{t} v_{i x}+\left(v_{i x} \partial_{x}+v_{i z} \partial_{z}\right) v_{i x}= \\
-\frac{e}{m_{i}} \partial_{x} \phi+\omega_{c i} v_{i y}-\frac{1}{m_{i} n_{i}} \partial_{x} p_{i} \\
\partial_{t} v_{i y}+\left(v_{i x} \partial_{x}+v_{i z} \partial_{z}\right) v_{i y}=-\omega_{c i} v_{i x}
\end{gathered}
$$

and

$$
\partial_{t} v_{i z}+\left(v_{i x} \partial_{x}+v_{i z} \partial_{z}\right) v_{i z}=-\frac{e}{m_{i}} \partial_{z} \phi-\frac{1}{m_{i} n_{i}} \partial_{z} p_{i} .
$$


From Poisson equation, the quasi-neutrality condition is defined as,

$$
n_{i}+n_{p} \simeq n_{e}
$$

The dispersion relation of ion-acoustic wave in a magnetized e-p-i plasma in the presence of hot ions is obtained by solving equations (3)-(11) algebraically, which yields,

$$
\begin{aligned}
(1-p) c_{s}^{2}\left\{\frac{k_{x}^{2}}{\left(\omega^{2}-\omega_{c i}^{2}\right)}+\frac{k_{z}^{2}}{\omega^{2}}\right\}= \\
(1+\alpha p)\left\{1-\frac{\frac{5}{3} \sigma c_{s}^{2} k_{x}^{2}}{\left(\omega^{2}-\omega_{c i}^{2}\right)}-\frac{\frac{5}{3} \sigma c_{s}^{2} k_{z}^{2}}{\omega^{2}}\right\} .
\end{aligned}
$$

In the limit of low frequency waves $\left(\omega \ll \omega_{c i}\right)$, the above dispersion relation reduces to,

$$
\omega^{2}=\frac{c_{s}^{2} k_{z}^{2}\left[(1-p)+\frac{5 \sigma}{3}(1+\alpha p)\right]}{\left[(1+\alpha p)+\left\{(1-p)+\frac{5 \sigma}{3}(1+\alpha p)\right\} \rho_{s}^{2} k_{x}^{2}\right]},
$$

where $\omega, k_{x}$ and $k_{z}$ are frequency and wave numbers along $x$ and $z$-direction, respectively. Furthermore $c_{s}=\left(\frac{T_{e}}{m_{i}}\right)^{\frac{1}{2}}$ is the ion-sound speed, $p=\frac{n_{p 0}}{n_{e 0}}$ is the ratio of positron and electron unperturbed densities, $\alpha=\frac{T_{e}}{T_{p}}$ is the ratio of electron and positron temperatures, $\sigma=\frac{T_{i}}{T_{e}}$ is the ratio of ion and electron temperatures and $\rho_{s}=\frac{c_{s}}{\omega_{c i}}$ is the ion Larmor radius at electron temperature. In the absence of hot ions i.e., $\sigma=0$, equation (13) gives the same dispersion relation as given in reference [16]. However, the limiting case of two component (e-i) plasma can be obtained from equation (13) by inserting $n_{p 0}=0$ (i.e., in the absence of positrons) and $\sigma=0$ (i.e., for cold ions case), which gives,

$$
\omega^{2}=\frac{c_{s}^{2} k_{z}^{2}}{\left(1+\rho_{s}^{2} k_{x}^{2}\right)} .
$$

Equation (14) is the same dispersion relation of IAW in a homogenous magnetized two components e-i plasma, which has already been studied by Yu et al. [17].

Equation (13) shows that finite ion temperature increases the ion acoustic speed and modifies the ion Larmor radius effects due to which wave dispersion term changes in magnetized e-p-i plasmas. A stable solitary structure is formed when nonlinear wave steepening balances with the wave dispersion of the ion-acoustic wave due to its ion Larmor radius effect. The wave dispersion effect is dependent on percentage presence of positrons and adiabatic ion temperature in magnetized e-p-i plasmas. Therefore it will be interesting to study the effects of finite ion temperature and positron concentration on the formation of solitary structures in magnetized e-p-i plasmas.

\section{Localized stationary solution}

For localized stationary solution, we define transformed coordinate $\xi$ such that,

$$
\xi=K_{x} x+K_{z} z-u t,
$$

where $u$ is the speed of the localized nonlinear structure moving with the frame and $K_{x}, K_{z}$ are the direction cosines along $x$-axis and $z$-axis, respectively. Furthermore $K_{x}^{2}+K_{z}^{2}=1$. The limitations of planar stationary solution is that the system of equations becomes stationary in the frame of reference and do not depends explicitly on time. Therefore, the time evolution of single stationary pulse (by applying the initial conditions) cannot be find from this method. The stationary planar solution is applicable only when an equilibrium is reached between nonlinear steepening and wave dispersion in the system and pulse becomes stable [20].

Now assuming that all the dependent variables are functions of $\xi$, the ion continuity given in equation (7) can be written as,

$$
v_{i x} \partial_{x}+v_{i z} \partial_{z}=M\left(1-\frac{1}{n_{i}}\right) \partial_{\xi} .
$$

Transforming equations (7)-(10) in terms of co-ordinate ' $\xi$ ' and using equation (16) in equations (8)-(10), we obtain the dimensionless nonlinear differential equation in terms of ion density $n_{i}$ as follows,

$$
\begin{aligned}
\frac{d}{d \xi}\left[\frac { 1 } { n _ { i } } \left\{\frac { d ^ { 2 } } { d \xi ^ { 2 } } \left(\frac{M^{2}}{2 n_{i}^{2}}+\right.\right.\right. & \left.\left.\left.\Phi+\frac{5}{2} \sigma n_{i}^{\frac{2}{3}}\right)+1\right\}\right]= \\
& -\frac{K_{z}^{2}}{M^{2}} n_{i} \frac{\partial \Phi}{\partial \xi}-\frac{5}{3} \frac{\sigma K_{z}^{2}}{M^{2}} n_{i}^{\frac{1}{3}} \frac{\partial n_{i}}{\partial \xi}
\end{aligned}
$$

where $\xi=\frac{\xi}{\rho_{s}}, M$ (Mach number) $=\frac{u}{c_{s}}, \Phi=\frac{e \phi}{T_{e}}, \sigma=\frac{T_{i}}{T_{e}}$ and $n_{j}=\frac{n_{j}}{n_{\alpha 0}}$ (where $j=\mathrm{e}, \mathrm{i}, \mathrm{p}$ ).

The quasi-neutrality condition gives,

$$
n_{i}=\frac{1}{(1-p)}\left(n_{e}-p n_{e}^{-\alpha}\right)
$$

where $\alpha=\frac{T_{e}}{T_{p}}$ and $p=\frac{n_{p 0}}{n_{e 0}}$. Equations (4) and (5) gives density relation between electrons and positrons such that $n_{p}=n_{e}^{-\alpha}$. It is to be noted here that above equation holds for $(0<p<1)$ in three component (e-p-i) plasmas.

Equation (4) in normalized form can be written as,

$$
\Phi=\ln n_{e} .
$$

Using equations (18) and (19) in equation (17) and then integrating it, we obtain differential equation in terms of $n_{e}$ as follows,

$$
\begin{array}{r}
\frac{d^{2}}{d \xi^{2}}\left[\frac{M^{2}(1-p)^{2}}{2\left(n_{e}-p n_{e}^{-\alpha}\right)^{2}}+\ln n_{e}+\frac{5}{2} \frac{\sigma\left(n_{e}-p n_{e}^{-\alpha}\right)^{\frac{2}{3}}}{(1-p)^{\frac{2}{3}}}\right]= \\
-1-\frac{\left(n_{e}-p n_{e}^{-\alpha}\right)}{(1-p)}\left\{\frac{K_{z}^{2}}{M^{2}} \frac{\left(n_{e}+\frac{p}{\alpha} n_{e}^{-\alpha}\right)}{(1-p)}\right. \\
-\frac{K_{z}^{2}}{M^{2}}\left(\frac{1+\frac{p}{\alpha}}{1-p}\right)-1-\frac{\sigma K_{z}^{2}}{M^{2}} \\
\left.+\frac{\sigma K_{z}^{2}}{M^{2}}\left(\frac{\left(n_{e}-p n_{e}^{-\alpha}\right)}{(1-p)}\right)^{\frac{5}{3}}\right\}
\end{array}
$$




$$
\begin{aligned}
V\left(n_{e}\right)= & {\left[\frac{1}{n_{e}}-\frac{M^{2}(1-p)^{2}\left(1+p \alpha n_{e}^{-\alpha-1}\right)}{\left(n_{e}-p n_{e}^{-\alpha}\right)^{3}}+\frac{5}{3} \frac{\sigma\left(1+p \alpha n_{e}^{-\alpha-1}\right)}{(1-p)^{\frac{2}{3}}\left(n_{e}-p n_{e}^{-\alpha}\right)^{\frac{1}{3}}}\right]^{-2} \times\left[\frac{M^{2}}{2}+\frac{K_{z}^{2}\left(1+\frac{p}{\alpha}\right)^{2}}{2 M^{2}(1-p)^{2}}+\frac{\left(1+\frac{p}{\alpha}\right)}{(1-p)}\right.} \\
& +\frac{\sigma K_{z}^{2}}{M^{2}} \frac{\left(1+\frac{p}{\alpha}\right)}{(1-p)}+\frac{5}{2} \sigma K_{z}^{2}-\frac{3}{2} \sigma+\frac{\sigma^{2} K_{z}^{2}}{2 M^{2}}+\left(1-K_{z}^{2}\right) \ln n_{e}+\frac{K_{z}^{2}\left(n_{e}+\frac{p}{\alpha} n_{e}^{-\alpha}\right)^{2}}{2 M^{2}(1-p)^{2}}-\left(n_{e}+\frac{p}{\alpha} n_{e}^{-\alpha}\right) \\
& \times\left\{\frac{1}{(1-p)}+\frac{K_{z}^{2}\left(1+\frac{p}{\alpha}\right)}{M^{2}(1-p)^{2}}+\frac{\sigma K_{z}^{2}}{M^{2}(1-p)}-\frac{K_{z}^{2}}{\left(n_{e}+\frac{p}{\alpha} n_{e}^{-\alpha}\right)}-\frac{\sigma K_{z}^{2}\left(n_{e}+\frac{p}{\alpha} n_{e}^{-\alpha}\right)^{\frac{5}{3}}}{M^{2}(1-p)^{\frac{8}{3}}}\right\}-\frac{1}{\left(n_{e}-p n_{e}^{-\alpha}\right)} \\
& \times\left\{M^{2}(1-p)-\frac{M^{2}(1-p)^{2}}{2\left(n_{e}-p n_{e}^{-\alpha}\right)}+K_{z}^{2}\left(1+\frac{p}{\alpha}\right)+\sigma K_{z}^{2}(1-p)\right\}-\left(n_{e}-p n_{e}^{-\alpha}\right)^{\frac{2}{3}}\left\{\frac{3}{2} \frac{\sigma K_{z}^{2}}{(1-p)^{\frac{2}{3}}}-\frac{5}{2} \frac{\sigma}{(1-p)^{\frac{2}{3}}}\right. \\
& \left.\left.+\frac{\sigma\left(n_{e}-p n_{e}^{-\alpha}\right)}{(1-p)^{\frac{5}{3}}}+\frac{\sigma K_{z}^{2}\left(1+\frac{p}{\alpha}\right)\left(n_{e}-p n_{e}^{-\alpha}\right)}{M^{2}(1-p)^{\frac{8}{3}}}+\frac{\sigma^{2} K_{z}^{2}\left(n_{e}-p n_{e}^{-\alpha}\right)}{M^{2}(1-p)^{\frac{5}{3}}}-\frac{1}{2} \frac{\sigma^{2} K_{z}^{2}\left(n_{e}-p n_{e}^{-\alpha}\right)^{\frac{8}{3}}}{M^{2}(1-p)^{\frac{10}{3}}}\right\}\right]
\end{aligned}
$$

where we have used the boundary conditions such that as $|\xi| \rightarrow \pm \infty, \frac{\partial^{2} n_{e}}{\partial \xi^{2}} \rightarrow 0, \frac{\partial n_{e}}{\partial \xi} \rightarrow 0$ and $n_{e} \rightarrow 1$.

Let us define

$$
R=\left[\frac{M^{2}(1-p)^{2}}{2\left(n_{e}-p n_{e}^{-\alpha}\right)^{2}}+\ln n_{e}+\frac{5}{2} \frac{\sigma\left(n_{e}-p n_{e}^{-\alpha}\right)^{\frac{2}{3}}}{(1-p)^{\frac{2}{3}}}\right] .
$$

Now multiplying by $\frac{\partial R}{\partial \xi}$ on both sides of equation (20) and after integrating once, we obtain,

$$
\frac{1}{2}\left(\frac{\partial n_{e}}{\partial \xi}\right)^{2}+V\left(n_{e}\right)=0
$$

where the Sagdeev potential is defined as,

$$
\text { see equation above, }
$$

we have used boundary conditions i.e., when $|\xi| \rightarrow \pm \infty$ then $\frac{\partial n_{e}}{\partial \xi} \rightarrow 0$ and $n_{e} \rightarrow 1$ to obtain above equation. Equation (21) is a well-known "energy integral" equation of an oscillating particle of a unit mass, with velocity $\left(\frac{\partial n_{e}}{\partial \xi}\right)$ at position $n_{e}$ in a nonlinear potential well $V\left(n_{e}\right)$.

The Sagdeev potential given in equation (21) gives the same results as obtained in reference [17], in limiting case of cold ions (i.e., $\sigma=0$ ) in magnetized e-p-i plasmas. However, the Sagdeev potential in two component (e-i) magnetized plasma can be obtained by substituting, $n_{p 0}=0$ and $\sigma=0$ in equation (21), which reduces to results obtained by $\mathrm{Yu}$ et al. [17]. In the absence of positrons, equation (21) also reduces to same results as obtained for e-i magnetized plasmas in the presence of adiabatic ions i.e., $\sigma \neq 0$ [19].

The conditions for the existence of localized solution of equation (21) require that (i) $V(1)=V(N)=$ $\left.\frac{\partial V}{\partial n_{e}}\right|_{n_{e}=1}=0$ and (ii) $\left.\frac{\partial^{2} V}{\partial \xi^{2}}\right|_{n_{e}=1}<0$ (where the fixed point at $n_{e}=1$ is unstable). Here $N$ is the point where the curve crosses the $n_{e}$-axis as shown in Figure 1 and it represents the maximum amplitude of the solitary wave.
From second condition, it is found that solitary structures are formed only in the subsonic region i.e., Mach number $(M<1)$.

\section{Numerical solutions}

In this section, some of the possible numerical solutions of equation (21) have been presented. The electron temperature and positron temperature are assumed to be same i.e., $\alpha=1$. In Figure 1, the Sagdeev potential curves for different values of ion temperature have been plotted for $p=0.2, K_{z}=0.8$ and $M=0.8$. It can be seen that the depth of Sagdeev potential curves decrease with the increase of adiabatic ion temperature in a magnetized e-p-i plasma. The corresponding electron density humps for the same parameters as given in Figure 1 are shown in Figure 2. It is evident that amplitude of the electron density hump is decreased with the increase of ion temperature in magnetized e-p-i plasmas. In Figure 3, the increase in the amplitude of the solitary pulse is shown with the increase in the positron concentration in magnetized e-p-i plasma with finite hot ion temperature i.e., $\sigma=0.02$ and $K_{z}=0.8$ and $M=0.8$. It is clear from the figure that width of the solitary pulse is significantly affected with positron concentration and solitary pulse get sharpened. In Figure 4, it is found that amplitude of the solitary pulse is increased with the increase in the obliqueness of the wave for $\sigma=0.02$ and $p=0.4$ and $M=0.6$. The potential distribution $\Phi$ at different values of $\left(n_{p 0} / n_{i 0}\right)$ i.e., equilibrium density ratio of positrons to ions, have been plotted using equation (19) as shown in Figure 5 where $n_{p 0} / n_{i 0}=p /(1-p)$ has been defined. It is clear from the figure that amplitude of the wave potential increases, while the width of the nonlinear structure decreases with the decrease of ions (or increase of positrons) in magnetized e-p-i plasmas. 


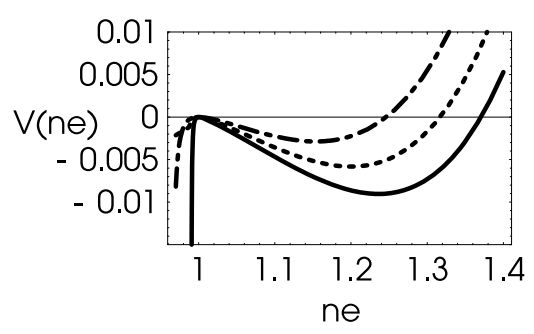

Fig. 1. The profiles of Sagdeev potential are shown in the absence and presence of hot ions i.e., $\sigma=0$ (solid curve), $\sigma=$ 0.02 (dashed curve) and $\sigma=0.05$ (broken curve) for $p=0.2$, $M=0.8$ and $K_{z}=0.8$ in a magnetized e-p-i plasma.

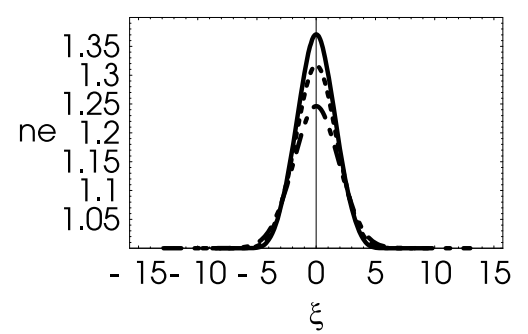

Fig. 2. The corresponding electron density humps are shown for the same parameters as given in Figure 1.

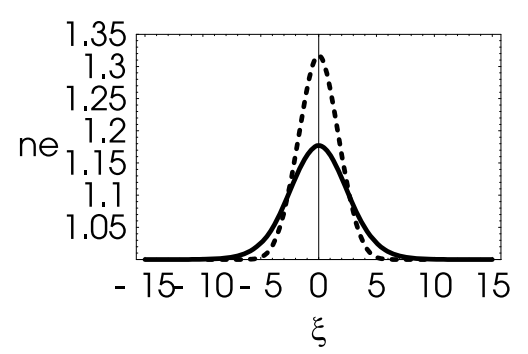

Fig. 3. The increase in the amplitude of electron density hump with the change in positron concentration in e-p-i plasmas is shown for $p=0.1$ (solid curve) and $p=0.2$ (dotted curve) with $\sigma=0.02, M=0.8$ and $K_{z}=0.8$.

\section{Conclusion}

We have studied the linear and nonlinear ion acoustic waves in the presence of adiabatically heated ions in magnetized e-p-i plasmas. The dispersion relation is also presented and modification of wave dispersion effects in the presence of adiabatic ions and different positron concentration are discussed. The Landau damping effects are ignored and assumption $c_{s} \gg v_{t i}$ (where $v_{t i}$ is the ion thermal velocity) remains valid for the consistency of the fluid model. The arbitrary amplitude ion acoustic solitary waves are studied with finite ion temperature in magnetized e-p-i plasmas by employing Sagdeev potential approach. It is found that the presence of hot ions decreases the wave amplitude significantly but there is a little change in the width of the nonlinear structure. However, the positron concentration increases the amplitude

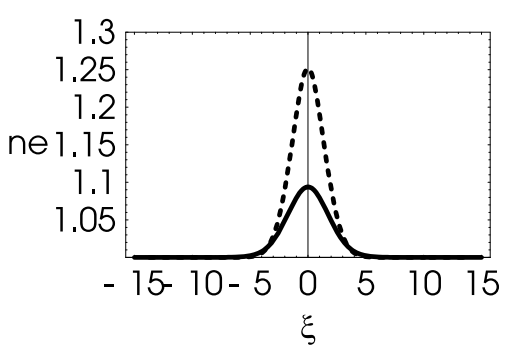

Fig. 4. The increase in the wave amplitude with the increase in obliqueness of the wave is shown for $K_{z}=0.8$ (solid curve) and $K_{z}=0.7$ (dotted curve) with $\sigma=0.02, p=0.4$ and $M=0.6$ in e-p-i plasmas.

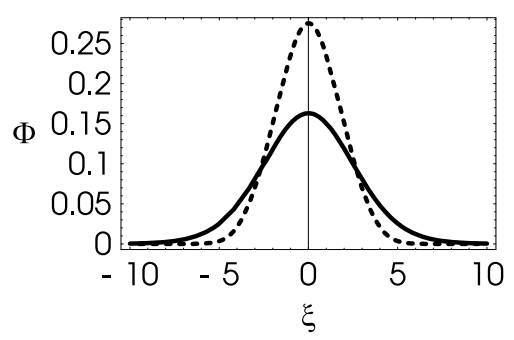

Fig. 5. The potential distribution $\Phi$ is shown for different values of equilibrium density ratio of positrons to ions i.e., $\frac{n_{p 0}}{n_{i 0}}=\frac{1}{9}$ at $p=0.1$ (shown in solid curve) and $\frac{n_{p 0}}{n_{i 0}}=\frac{1}{4}$ at $p=0.2$ (shown in dotted curve) for $\sigma=0.02, M=0.8$ and $K_{z}=0.8$ in magnetized e-p-i plasmas.

and decreases the width of the nonlinear structure in magnetized in e-p-i plasmas. Therefore, solitary structure gets sharpened with the increase of positron concentration in a magnetized multicomponent plasma. It is also found that the amplitude of the nonlinear structure is increased with the increase of obliqueness of the wave in e-p-i plasmas. Our findings are general and may be applicable to astrophysical plasma situations such as polar cups region of pulsars and around active galactic nuclei where magnetized e-p-i plasma with adiabatic ions can exist.

\section{References}

1. P. Goldreich, W.H. Julian, Astrophys. J. 157, 869 (1969)

2. F.C. Michel, Rev. Mod. Phys. 54, 1 (1982)

3. H.R. Miller, P.J. Witter, Active Galactic Nuclei (SpringerVerlag, Berlin, 1987), p. 202

4. V.I. Berezhiani, M.Y. El-Ashry, U.A. Mofiz, Phys. Rev. E 50, 448 (1994)

5. V.I. Berezhiani, S.M. Mahajan, Phys. Rev. Lett. 73, 1110 (1994)

6. J. Zhao, J.I. Sakai, K.I. Nishikawa, T. Neubert, Phys. Plasmas 1, 4114 (1994)

7. G.P. Zank, R.G. Gareaves, Phys. Rev. E 51, 6079 (1995); S.J. Gilbert, D.H.E. Dubin, R.G. Greaves, C.M. Surko, Phys. Plasmas 8, 4982 (2001)

8. N. Iwamoto, Phys. Rev. E 39, 604 (1993) 
9. C.M. Surko et al., Rev. Sci. Instrum. 57, 1862 (1986)

10. C.M. Surko, T. Murphy, Phys. Fluids B 2, 1372 (1990)

11. V.I. Karpman, Physica Scripta 11, 271 (1975)

12. S.I. Popel, S.V. Vladimirov, P.K. Shukla, Phys. Plasmas 2, 716 (1995)

13. Y.N. Nejoh, Australian J. Phys. 50, 309 (1997)

14. Y.N. Nejoh, Phys. Plasmas 3, 1447 (1996)

15. H. Hasegawa, S. Irie, S. Usami, Y. Ohsawa, Phys. Plasmas 9, $2549(2002)$
16. S. Mahmood, A. Mushtaq, H. Saleem, New J. Phys. 5, 28 (2003)

17. M.Y. Yu, P.K. Shukla, S. Bujarbarua, Phys. Fluids 23, 2146 (1980)

18. P.K. Shukla, M.Y. Yu, J. Math. Phys. 19, 2506 (1978)

19. M.K. Kalita, S. Bujarbarua, J. Phys. A 16, 439 (1983)

20. R.A. Treumann, W. Baumjohann, Advanced Space Plasma Physics (Imperial College, London, 1997), Chap. 10 